\title{
TRANSFORMATIONS BY FUNCTIONS IN SOBOLEV SPACES AND LOWER SEMICONTINUITY FOR PARAMETRIC VARIATIONAL PROBLEMS
}

\author{
BY M. MARCUS AND V. J. MIZEL \\ Communicated by James Serrin, December 18, 1972
}

Introduction. In this work we are concerned with integrals of the form

$$
\int_{\Omega} f\left(x, \varphi(x), \varphi_{x}(x)\right) d \mathscr{L}_{m}(x)
$$

where $\Omega \subset R_{m}$ is a bounded domain, $\mathscr{L}_{m}$ denotes $m$-dimensional Lebesgue measure, $\varphi=\left(\varphi^{1}, \ldots, \varphi^{n}\right)^{\text {tr }}$ is a continuous transformation from $\Omega$ to $R_{n}(m \leqq n)$ belonging to some Sobolev space $W_{p}^{1, \text { loc }}(\Omega)$, and $\varphi_{x}$ is the (almost everywhere defined) matrix function $\left(\partial \varphi^{i} / \partial x_{j}\right)$. Here $f$ is a realvalued function on $\Omega \times R_{n} \times M_{n \times m}$, where $M_{n \times m}$ is the space of $n \times m$ matrices. Such integrals arise in the analysis of various variational problems. The success of the analysis, by direct methods, of nonparametric variational problems, where it is appropriate to make direct convexity hypotheses on $f(x, z, \cdot)$, is well known. In contrast, the study of parametric variational problems by these methods has been less successful. In this latter class of problems, which includes Plateau's problem, the fact that the integral has special behavior under transformation of independent variables implies that the integrand cannot possess the strong type of convexity properties referred to above. Only for the case $m=2$ has there been available a lower semicontinuity result for such problems, in what would seem to be their natural context, namely with $\varphi$ continuous and belonging to $W_{m}^{1}(\Omega)^{n}$ and with sequences $\left\{\varphi^{k}\right\}$ converging uniformly to $\varphi$. An important example due to Besicovitch [1] has indicated that the situation here is a very delicate one. For $m>2$, a result of this type was obtained by Morrey [7, Theorem 9.2.1] under the additional assumption that $\varphi$ is locally Lipschitz in $\Omega$.

The present note provides, under natural hypotheses for parametric integrands $[7$, p. 356], a lower semicontinuity result for the general case of $\varphi \in W_{m}^{1}(\Omega)^{n} \cap C(\Omega)^{n}, m \geqq 2$. The result is obtained by techniques due to McShane [5] and Morrey [7], combined with a measure-geometric estimate for mappings $\varphi \in W_{p}^{1}(\Omega)^{n} \cap C(\Omega)^{n}, p>m$. This estimate, together with a few additional results concerning mappings $\varphi$ of this type, is presented in the first part of this note.

AMS (MOS) subject classifications (1970). Primary 28A75, 49F22; Secondary 26A57, 49F25, 46E 35 .

Key words and phrases. Change of variables formula, Sard lemma, Sobolev spaces, parametric variational problem, lower semicontinuity, Lebesgue-Fréchet surface area. 
Among these results we mention an elementary change of variables formula for integrals involving mappings $\varphi \in W_{p}^{1}(\Omega)^{n} \circ \cap C(\Omega)^{n}, p>m$, and a related generalization of Sard's lemma. The change of variables formula is based on a theorem of Federer [3] and extends a result of Rado and Reichelderfer [8].

Complete proofs and related results will appear elsewhere.

1. Transformations in $W_{p}^{1}(\Omega)^{n}, p>m$. We adopt the convention that whenever $\varphi$ belongs to $W_{p}^{1}(\Omega)^{n}, p>m$, it is taken to be the continuous representative of its equivalence class. The existence of such a representative is ensured by the embedding theorem.

Our first result is an estimate for the $\mathscr{H}_{m}$-measure of $\varphi$-images of measurable subsets of $\Omega$, where $\mathscr{H}_{m}$ denotes the $m$-dimensional Hausdorff measure in $R_{n}$.

THEOREM 1. Suppose $\varphi: \Omega \rightarrow R_{n}$ belongs to $W_{p}^{1}(\Omega)^{n}, p>m$. Then $S=\varphi(\Omega)$ is of finite $\mathscr{H}_{m}$-measure and for every $\mathscr{L}_{m}$-measurable subset $A \subset \Omega$ one has

$$
\mathscr{H}_{m}(\varphi(A)) \leqq C(m, p) \mathscr{L}_{m}(A)^{1-m / p}\left[\int_{A}\left|\varphi_{x}(x)\right|^{p} d \mathscr{L}_{m}(x)\right]^{m / p} .
$$

Remark. The requirement $p>m$ is essential since Besicovitch ([1], see also [7, p. 352]) has provided an example, with $m=2$, of a continuous $\varphi$ in $W_{2}^{1}(\Omega)$ which satisfies $\mathscr{H}_{3}(\varphi(\Omega))>0$ !

Corollary 1. If $N \subset \Omega$ is $\mathscr{L}_{m}$-null, then $\varphi(N)$ is $\mathscr{H}_{m}$-null.

CoRollary 2. If $A \subset \Omega$ is $\mathscr{L}_{m}$-measurable, then $\varphi(A)$ is $\mathscr{H}_{m}$-measurable.

The proof of Theorem 1 can be reduced to the case where $A$ is open. We partition $A$ into (half open) cubes $\left\{Q^{j}\right\}_{j \geqq 1}$. By using a variant of Poincaré's lemma for $W_{p}^{1}(\Omega)($ e.g., $[7$, p. 83]) we obtain for each of these cubes the estimate

$$
\int_{Q}\left|\varphi^{i}(x)-\bar{\varphi}^{i}\right|^{p} d \mathscr{L}_{m}(x) \leqq C_{Q} \int_{Q}\left|\varphi_{x}^{i}(x)\right|^{p} d \mathscr{L}_{m}(x), \quad i=1, \ldots, n,
$$

where $\bar{\varphi}^{i}=\bar{\varphi}^{i}(Q)$ is the mean value of $\varphi^{i}$ over $Q$. Combining this with the Sobolev embedding theorem we obtain

$$
\max _{Q}\left|\varphi^{i}-\bar{\varphi}^{i}\right| \leqq C_{Q}^{\prime}\left\|\nabla \varphi^{i}\right\|_{L^{p}(Q)}, \quad i=1, \ldots, n,
$$

where it is easily seen that $C_{Q}^{\prime}$ has the form

$$
C_{Q}^{\prime}=C^{\prime}(m, p) \cdot r^{1-m / p},
$$

$r$ being the edge length of $Q$. Using the estimates (1.3), (1.4), for each $i=1, \ldots, n$, we deduce that $\varphi\left(Q^{j}\right)$ is contained in the $R_{n}$-cube centered 
at $\bar{\varphi}\left(Q^{j}\right)=\left(\bar{\varphi}^{1}\left(Q^{j}\right), \ldots, \bar{\varphi}^{n}\left(Q^{j}\right)\right)$ and having edges of length

$$
l_{j}=2 C^{\prime}(m, p)\left(r_{j}\right)^{1-m / p}\left\|\varphi_{x}\right\|_{L^{p}\left(Q^{j}\right)^{n}},
$$

where $r_{j}$ is the edge length of $Q^{j}$. Thus $\varphi\left(Q^{j}\right)$ is contained in a ball $B^{j}$ of diameter

$$
d_{j}=2 m^{1 / 2} C^{\prime}(m, p)\left(r_{j}\right)^{1-m / p}\left\|\varphi_{x}\right\|_{L^{p}\left(Q^{j}\right)^{n}}, \quad j \geqq 1 .
$$

From (1.5) it follows, since the absolute continuity of $\int\left|\varphi_{x}\right|^{p}$ ensures that for the $\left\{Q^{j}\right\}$ taken sufficiently small the $\left\{B^{j}\right\}$ will be of arbitrarily small diameter, that the outer $\mathscr{H}_{m}$-measure of the set $\varphi(A)=\bigcup_{j} \varphi\left(Q^{j}\right)$ satisfies

$$
\begin{aligned}
\mathscr{H}_{m}(\varphi(A)) & \leqq C(m, p) \sum_{j}\left(r_{j}\right)^{m(1-m / p)}\left\|\varphi_{x}\right\|_{L^{p}\left(Q^{j}\right)^{n}}^{m} \\
& \leqq C(m, p)\left[\sum_{j}\left(r_{j}\right)^{m}\right]^{1-m / p}\left[\sum_{j} \int_{Q_{j}}\left|\varphi_{x}(x)\right|^{p} d \mathscr{L}_{m}(x)\right]^{m / p} \\
& =C(m, p) \mathscr{L}_{m}(A)^{1-m / p}\left[\int_{A}\left|\varphi_{x}(x)\right|^{p} d \mathscr{L}_{m}(x)\right]^{m / p}
\end{aligned}
$$

For our next result we introduce the following notation (see [3]). We set, for $\varphi$ as above, $A \subset \Omega$ and $y \in R_{n}, N(\varphi \mid A, y)=$ cardinality $\left\{\varphi^{-1}(y) \cap A\right\}$.

In addition, for any $P \in M_{n \times m}$ we denote $\sigma_{m}(P)=\left(\sum_{\lambda}\left(X_{\lambda}\right)^{2}\right)^{1 / 2}$, where $\left\{X_{\lambda}\right\}$ are the $m \times m$ minors of the matrix $P$. Note that by a result of Morrey [6] (see also Gagliardo [4]) $\varphi_{x}$, and hence $J_{m} \varphi \equiv \sigma_{m}\left(\varphi_{x}\right)$, is defined $\mathscr{L}_{m}$-a.e. in $\Omega$, where the partial derivatives are taken in the classical sense.

TheOrem 2 (Change of Variables). Let $\varphi$ be as in Theorem 1 and let $g: \varphi(\Omega) \rightarrow R$ be an $\mathscr{H}_{m}$-measurable function. If $A$ is an $\mathscr{L}_{m}$-measurable subset of $\Omega$, the formula

$$
\int_{\varphi(A)} g(y) N(\varphi \mid A, y) d \mathscr{H}_{m}(y)=\int_{A} g(\varphi(x)) J_{m} \varphi(x) d \mathscr{L}_{m}(x)
$$

is valid, whenever one of the two sides is meaningful.

Remark. For the case $n=m=2$, this result was proved by Rado and Reichelderfer [8, p. 438]. Actually, it is easily seen that their proof also gives the result for the case $n=m \geqq 2$.

When $n \geqq m$, formula (1.7) was obtained by Federer [3, pp. 241 and 244] under the following assumptions:

(a) $\varphi$ is continuous and the (classical) partial derivatives $\partial \varphi / \partial x_{i}$ $(i=1, \ldots, m)$ exist $\mathscr{L}_{m}$-a.e. in $\Omega$;

(b) $\varphi$ satisfies the inequality

$$
\limsup _{x^{\prime} \rightarrow x}\left|\varphi\left(x^{\prime}\right)-\varphi(x)\right| \cdot\left|x^{\prime}-x\right|^{-1}<\infty,
$$

at every point $x$ in $A$. 
ProOF. By a result of Calderón [2], the transformation $\varphi$ satisfies (1.8) at $\mathscr{L}_{m}$-almost every point $x$ in $\Omega$. Denote by $N$ the null subset of $\Omega$ where (1.8) does not hold.

Let $A$ be an $\mathscr{L}_{m}$-measurable subset of $\Omega$ and set $A_{1}=A-N$ and $A_{2}=A \cap N$.

By the result of Federer mentioned above, (1.7) is valid if $A$ is replaced by $A_{1}$. But, by Corollary $1, \varphi\left(A_{2}\right)$ is an $\mathscr{H}_{m}$-null set in $R_{n}$. Hence formula (1.7) holds.

Corollary 3 (Generalized SARD's lemma). Let $\boldsymbol{\varphi}$ be as in the theorem. Then for every $\mathscr{L}_{m}$-measurable subset $A \subset \Omega$ one has

$$
\mathscr{H}_{m}(\varphi(A)) \leqq \int_{A} J_{m} \varphi(x) d \mathscr{L}_{m}(x)
$$

In particular, if $A_{0}=\left\{x \mid \operatorname{rank}\left(\varphi_{x}\right)<m\right\}$ then $\mathscr{H}_{m}\left(\varphi\left(A_{0}\right)\right)=0$.

COROllaRY 4. If $A \subset \Omega$ is such that $\mathscr{H}_{m}(\varphi(A))=0$, then

$$
\operatorname{rank}\left(\varphi_{x}(x)\right)<m \text { for a.e. } x \in A \text {. }
$$

REMARK. Notice that the right side of equation (1.9) is finite even for $p=m$, but the example of Besicovitch mentioned above shows that the inequality can fail in this case.

2. Semicontinuity results for $W_{m}^{1}(\Omega)^{n}$. We now apply the preceding results to parametric variational problems, following the basic scheme in [?, Chapter 9]. Hereafter we deal with integrands $(0.1)$ which satisfy the following conditions $[7$, p. 356]:

(2.1) (a) $f$ is independent of $x \in \Omega$ and $m \leqq n$;

(b) $f: R_{n} \times M_{n \times m} \rightarrow R$ is continuous everywhere and of class $C^{1}$ on the open set $U=\{(z, P): \operatorname{rank}(P)=m\}$.

(2.2) $f(z, P A)=(\operatorname{det} A) f(z, P)$ for every $m \times m$ matrix $A$ such that $\operatorname{det} A>0$.

(2.3) $f(z, P)=F\left(z,\left(X_{\lambda}\right)\right)$, where $F(z, \cdot)$ is a convex function in the $m \times m$ minors $\left\{X_{\lambda}\right\}$ of $P$ :

$$
f\left(z^{0}, P\right)=F\left(z^{0},\left(X_{\lambda}\right)\right) \geqq F\left(z^{0},\left(X_{\lambda}^{0}\right)\right)+\sum_{\lambda} a_{\lambda}^{0}\left(X_{\lambda}-X_{\lambda}^{0}\right) .
$$

Moreover, if $\left(z^{0}, P^{0}\right) \in U$, the coordinates $\left\{a_{\lambda}^{0}\right\}$ of the support plane to $F\left(z^{0}, \cdot\right)$ at $\left(X_{\lambda}^{0}\right)$ satisfy certain additional requirements by which they are uniquely determined (see [7, p. 354]). If $\left(z^{0}, P^{0}\right) \notin U$ (in which case $X_{\lambda}^{0}=0$ for all $\lambda$, and $f\left(z^{0}, P^{0}\right)=0$ by $(2.2)$ ), the coordinates $\left\{a_{\lambda}^{0}\right\}$ are not uniquely determined.

(2.4) $f(z, P) \geqq c \sigma_{m}(P)$, for some $c>0$.

REMARKS. Conditions (2.1)(a), (2.2) are related to the invariant nature of the integral under diffeomorphisms of $\Omega$, while (2.3) implies the strong 
quasi-convexity of $f$ with respect to $P$ [7, Theorem 4.4.8]. Actually, if $n=m+1$, the convexity of $F$ with respect to $\left(X_{\lambda}\right)$ is equivalent to the strong quasi-convexity of $f$ with respect to $P$ [7, Theorem 4.4.10].

The particular case $f(z, P)=\sigma_{m}(P)$, which occurs in the Plateau problem, is easily seen to satisfy $(2.1)-(2.4)$

We now give our basic semicontinuity result.

THEOREM 3 (Lower SEMICONTINUITY). Let $f$ satisfy (2.1)-(2.4). Suppose that $\left\{\varphi_{k}\right\}$ and $\varphi$ belong to $W_{m}^{1, \text { loc }}(\Omega)^{n} \cap C(\Omega)^{n}$ and $\varphi_{k} \rightarrow \varphi$ locally uniformly on $\Omega$. Then

$$
\int_{\Omega} f\left(\varphi(x), \varphi_{x}(x)\right) d \mathscr{L}_{m}(x) \leqq \liminf \int_{\Omega} f\left(\varphi_{k}(x), \varphi_{k, x}(x)\right) d \mathscr{L}_{m}(x) .
$$

Observe that one can restrict attention to cases in which the right side of (2.5) is finite. It is then sufficient to verify (2.5) for subdomains $\Omega^{\prime}$ for which $\overline{\Omega^{\prime}} \subset \Omega$. The proof utilizes the following lemmas. We follow [7] in denoting the Jacobian $\operatorname{det}\left(\psi_{x}\right)$ by $\partial \psi / \partial x$.

LeMmA 1. If $\psi \in W_{m}^{1, \text { loc }}(\Omega)^{m} \cap C(\Omega)^{m}$ then there exist $\mathscr{L}_{1}$-null sets $Z_{i}$, $i=1, \ldots, m$, such that if $R$ is any closed cell in $\Omega$ none of whose faces lie on one of the planes $x^{i}=c^{i}$ where $c^{i} \in Z_{i}$, then

$$
\int_{R} \frac{\partial \psi}{\partial x}(x) d \mathscr{L}_{m}(x)=\int_{R_{m}} o[y, \psi(\partial R)] d \mathscr{L}_{m}(y),
$$

where $o[y, \psi(\partial R)]$ denotes the order at $y$ of the map $\psi$ on $\partial R$.

LEMMA 2. Let $\psi$ be as in Lemma 1, and suppose $R$ is a cell for which (2.6) holds. Let $\left\{\psi_{k}\right\}$ satisfy the same conditions as $\psi$ and suppose $\psi_{k} \rightarrow \psi$ uniformly on $R$. Then there are measurable subsets $V_{k} \subset R$ such that

$$
\int_{V_{k}} \frac{\partial \psi_{k}}{\partial x}(x) d \mathscr{L}_{m}(x) \rightarrow \int_{R} \frac{\partial \psi}{\partial x}(x) d \mathscr{L}_{m}(x) .
$$

In the proof of Lemma 1 we utilize Theorem 1 together with a characterization of Sobolev spaces due to Morrey [6] and Gagliardo [4] to show that almost all hyperplanes $x^{i}=$ const, $i=1, \ldots, m$, are mapped by $\psi$ into $\mathscr{H}_{m}$-null sets. Lemma 2 is an extension along the lines of [7] of a result due to McShane [5].

Our final result involves an extension of the functional $I_{f}$, given on $W_{m}^{1}(\Omega)^{n} \cap C(\Omega)^{n}$ by

$$
I_{f}(\varphi, \Omega)=\int_{\Omega} f\left(\varphi(x), \varphi_{x}(x)\right) d \mathscr{L}_{m}(x),
$$

to all of $C(\Omega)^{n}$ along the lines of the definition of Lebesgue-Fréchet area (see Serrin [9], and [7, p. 353]). 
Definition. For $f$ satisfying (2.1)-(2.4) and $\varphi \in C(\Omega)^{n}$ define

$$
\mathscr{I}_{f}(\varphi, \Omega)=\inf \left(\liminf _{k \rightarrow \infty} \int_{\Omega} f\left(\varphi_{k}(x), \varphi_{k, x}(x)\right) d \mathscr{L}_{m}(x)\right),
$$

the infimum being taken over all sequences $\left\{\varphi_{k}\right\}_{k \geqq 1}$ of $C^{1}(\Omega)^{n}$-transformations which converge locally uniformly to $\varphi$.

The following theorem, which resolves a question raised by Morrey $[7$, p. 400], is a consequence of the lower semicontinuity result.

THEOREM 4. Let $f$ satisfy (2.1)-(2.4). If $\varphi \in W_{m}^{1}(\Omega)^{n} \cap C(\Omega)^{n}$ then

$$
\mathscr{I}_{f}(\varphi, \Omega)=\int_{\Omega} f\left(\varphi(x), \varphi_{x}(x)\right) d \mathscr{L}_{m}(x) .
$$

REMARK. The results of this section actually hold under the following more general assumptions on $\varphi$ :

where

$$
\varphi \in W_{p_{1}}^{1}(\Omega) \times W_{p_{2}}^{1}(\Omega) \times \cdots \times W_{p_{n}}^{1}(\Omega) \cap C(\Omega)^{n},
$$

(a) $p_{i}>m-1, i=1, \ldots, n$,

(b) $1 / p_{i_{1}}+\cdots+1 / p_{i_{m}} \leqq 1$ whenever $1 \leqq i_{1}<\cdots<i_{m} \leqq n$.

ACKNOWLEDGEMENTS. The work of one of us (VJM) was partially supported by the National Science Foundation under Grant GP28377 and took place during the tenure of a Visiting Professorship at the Technion. He wishes to acknowledge the warm hospitality of the Technion Mathematics Department during his stay.

Professor $\mathrm{H}$. Brezis brought to our attention the problem of developing a change of variables result such as Theorem 2 .

\section{BIBLIOGRAPHY}

1. A. S. Besicovitch, Parametric surfaces, Bull. Amer. Math. Soc. 56 (1950), 288-296. MR 12, 168.

2. A. P. Calderón, On the differentiability of absolutely continuous functions, Riv. Mat. Univ. Parma 2 (1951), 203-213. MR 13, 544.

3. H. Federer, Geometric measure theory, Die Grundlehren der Math. Wissenschaften, Band 153, Springer-Verlag, New York, 1969. MR 41 \# 1976.

4. E. Gagliardo, Proprietà di alcune classi di funzioni in più variabili, Ricerche Mat. 7 (1958), 102-137. MR 21 \# 1526.

5. E. J. McShane, On the semi-continuity of doublc integrals in the calculus of variations, Ann. of Math. 33 (1932), 460-484.

6. C. B. Morrey, Functions of several variables and absolute continuity. II, Duke Math. J. 6 (1940), 187-215. MR 1, 209.

7. 1 , Multiple integrals in the calculus of variations, Die Grundlehren der Math. Wissenschaften, Band 130, Springer-Verlag, 1966. MR 34 \# 2380.

8. T. Rado and P. V. Reichelderfer, Continuous transformations in analysis. With an introduction to algebraic topology, Die Grundlehren der Math. Wissenschaften, Band 75, Springer-Verlag, 1955. MR 18, 115.

9. J. Serrin, On the definitions and properties of certain variational integrals, Trans. Amer. Math. Soc. 101 (1961), 139-167. MR 25 \#1466.

Department of Mathematics, Technion, IsRael Institute of Technology, Haifa, ISRAEL

Department of Mathematics, Carnegie-Mellon University, Pittsburgh, PennsylVANIA 15213 